Pacific Journal of Mathematic 


\title{
PRECOMPACT AND COLLECTIVELY SEMI-PRECOMPACT SETS OF SEMI-PRECOMPACT CONTINUOUS LINEAR OPERATORS
}

\author{
ANDREW S. GeUe
}

\begin{abstract}
A mapping $f$ from a set $B$ into a uniform space $(Y, \mathscr{V})$ is said to be precompact if and only if its range $f(B)=$ $\{f(b): b \in B\}$ is a precompact subset of $Y$. The precompact subsets of $\mathscr{K}(B, Y)$, the set of all precompact mappings from $B$ into $Y$ with its natural topology of uniform convergence, are characterized by an Ascoli-Arzelà theorem using the notion of equal variation.

A linear operator $T: X \rightarrow Y$, where $X$ and $Y$ are topological vector spaces, is said to be semi-precompact if $T(B)$ is precompact for every bounded subset $B$ of $X$. Let $\mathscr{L}_{\mathrm{b}}[X, Y]$ denote the set of all continuous linear operators from $X$ into $Y$ with the topology of uniform convergence on bounded subsets of $X$. Let $\mathscr{K}_{\mathfrak{b}}[X, Y]$ denote the subspace of $\mathscr{L}_{\mathfrak{b}}[X, Y]$ consisting of the semi-precompact continuous linear operators with the induced topology. The precompact subsets of $\mathscr{K}_{\tilde{b}}[X, Y]$ are characterized. A generalized Schauder's theorem for locally convex Hausdorff spaces is obtained. A subset $\mathscr{C}$ of $\mathscr{L}[X, Y]$ is said to be collectively semi-precompact if $\mathscr{H}(B)=\{H(b): H \in \mathscr{H}, b \in B\}$ is precompact for every bounded subset $B$ of $X$. Let $X$ and $Y$ be locally convex Hausdorff spaces with $Y$ infrabarrelled. In $\S 5$ the precompact sets of semi-precompact linear operators in $\mathscr{L}_{\mathfrak{b}}[X, Y]$ are characterized in terms of the concept of collective semi-precompactness of the sets and certain properties of the set of adjoint operators.
\end{abstract}

1. Introduction. Let $X$ and $Y$ be topological vector spaces over the field of complex numbers $C$ and $\mathscr{L}[X, Y]$ the set of continuous linear operators from $X$ into $Y$. For a subset $\mathscr{C} \subset \mathscr{L}[X, Y]$ and a subset $B$ of $X$, let $\mathscr{H}(B)=\{H(b): H \in \mathscr{C}, b \in B\}$.

Definition 1.1. A linear operator $T: X \rightarrow Y$ is said to be precompact (compact) if there exists a neighborhood $V$ of zero in $X$ such that $T(V)$ is precompact (relatively compact). A linear operator $T: X \rightarrow Y$ is said to be semi-precompact (semi-compact) if $T(B)$ is precompact (relatively compact) for every bounded subset $B$ of $X$.

The latter terminology is that of Deshpande and Joshi [14] and coincides with the term "boundedly precompact" used by Ringrose [27]. Clearly, precompactness of an operator is a much stronger 
condition than semi-precompactness, unless $X$ has a bounded neighborhood of zero. A precompact operator is always continuous, but this is not the situation for a semi-precompact operator unless we assume $X$ bornological and $Y$ locally convex [18, Proposition 1(a), p. 220]. However, we shall always work with continuous semi-precompact operators in this paper and thus avoid the problems otherwise encountered.

Definition 1.2. A subset $\mathscr{H} \subset \mathscr{L}[X, Y]$ is said to be collectively precompact (collectively compact) if there exists a neighborhood $V$ of zero in $X$ such that $\mathscr{H}(V)$ is precompact (relatively compact). A subset $\mathscr{H} \subset \mathscr{L}[X, Y]$ is said to be collectively semi-precompact (collectively semi-compact) if $\mathscr{H}(B)$ is precompact (relatively compact) for every bounded subset $B$ of $X$.

The latter concept is again due to Deshpande and Joshi [14].

Anselone and Moore [5] introduced the concept of collectively compact sets of linear operators on normed linear spaces in connection with approximate solutions of integral and operator equations and this material, together with much other work in this area, appears in Anselone [4]. Anselone and Palmer [6, 7, 8] studied the general properties of such sets of operators, again in normed linear spaces. DePree and Higgins [11] and Deshpande and Joshi [14] generalized some of the theorems of Anselone and Palmer to the topological vector space situation. More insight into the idea of collectively compact sets of linear operators is given in the characterizations of DePree and Klein [12] (or Klein [22, Chapter I]), where the set is factored through a Banach space via an equicontinuous collection and a compact operator. DePree and Klein [13] (or Klein [22, Chapter II]) have applied the concept of collectively compact sets of linear operators to semi-groups of compact linear operators. However, a characterization of precompact sets of precompact linear operators via the adjoint operators has not yet been obtained beyond the Banach space case. Palmer [25] proved that for Banach spaces $X$ and $Y$, a collectively compact subset $\mathscr{H} \subset \mathscr{L}[X, Y]$ is precompact in the uniform operator topology if and only if $\mathscr{H}^{\prime}$, the set of adjoint operators, is collectively compact. Anselone conjectured this in [2] and also proved it for special Banach spaces $X$ and $Y$. In [3], Anselone also gave a different proof of the general result of Palmer [25]. We notice that Schauder's theorem for compact operators is implicit in this result.

The problem in dealing with general precompact operators is that no corresponding Schauder theorem holds even when $X$ and $Y$ are locally convex spaces. This result was stated in Köthe [23], 
where he says that Grothendieck exhibited an example of a Fréchet space for which Schauder's theorem does not hold. However, when dealing with semi-precompact operators a corresponding Schauder theorem does hold provided $X$ and $Y$ are locally convex Hausdorff spaces with $Y$ infrabarrelled [21, Problem 21D, p. 208] and easily proved using Grothendieck [17, Lemma 2, p. 132]. The theorem also follows easily from a more general theorem in $\S 4$ of this paper. The condition that $Y$ be infrabarrelled is very essential for it turns out that it is a necessary and sufficient condition for the adjoint mapping to be continuous ( $\$ 5$, Lemma 5.3). To obtain results similar to Palmer [25] we must be able to say that a precompact set of precompact operators is collectively precompact. This is true in general Banach spaces [6, Theorem 2.5, p. 419], but it is not true for general locally convex spaces as shown by the counterexample of DePree and Higgins [11, Example 3.8, p. 369]. However, when we consider locally convex Hausdorff spaces and the concepts of semi-precompactness and collective semi-precompactness of operators, Palmer's results carry over as shown in $\$ 5$.

The problem of proving that a set of precompact operators is collectively precompact is that in general each operator is defined to be precompact only on a particular neighborhood of zero in $X$. To find a single neighborhood $V$ of zero in $X$ such that every operator in the set is precompact on this neighborhood is a very difficult task without even proving the set collectively precompact on this neighborhood. The problem is avoided if $X$ is a normed space as we only need speak of a single neighborhood of zero, namely the unit ball. It is also avoided when $X$ has a bounded neighborhood of zero [11, Theorem 3.6, p. 368], as any precompact operator will be precompact on this neighborhood. Anyway, if $X$ is also locally convex Hausdorff, then it is normed [28, Theorem 1, p. 45]. The problems above no longer arise when considering collective semiprecompactness, as we look at what the operators do to each bounded subset of $X$.

Most of the results of DePree and Higgins [11] and Deshpande and Joshi [14], which generalize $\S 2$ of Anselone and Palmer [6], carry over quite easily to the collectively semi-precompact case and will not be considered here.

The proofs of most of the theorems are based on generalizations of results in Vala [32]. The idea of equal variation, as it is called by Vala [32] (and inherent in the works of Kakutani [19], Bartle [9], Dunford and Schwartz [15, Theorem 6, p. 260] and Poppe [26]), plays a most important role in the characterizations via an AscoliArzelà type theorem. The generalized Schauder theorem for Banach spaces [32, Theorem 3, p. 6] is proved for locally convex Hausdorff 
spaces and the proof employs the ideas of $\S \S 2$ and 3. Part of this Schauder type theorem was also proved for the Banach space case by Alexander [1] and Bonsall [10] in a Banach algebra setting.

2. Preliminaries. Let $B$ be an arbitrary set and $(Y, \mathscr{Y})$ a uniform space.

Definition 2.1. A mapping $f: B \rightarrow Y$ is said to be precompact (compact) if the set $f(B)=\{f(b): b \in B\}$ is precompact (relatively compact) in $Y$. Let $\mathscr{K}(B, Y)$ denote the set of all precompact mappings from $B$ into $Y$.

Obviously every compact mapping is precompact and the converse holds if $Y$ is complete, as then every precompact set is relatively compact. If $Y$ is a topological vector space, quasi-completeness is a sufficient condition for the converse to hold.

When $Y$ is a metric space with metric $d$, there is a natural metric we can define on $\mathscr{K}(B, Y)[32$, p. 3], namely $\rho$, where

$$
\rho(f, g)=\sup _{b \in B} d(f(b), g(b))
$$

for $f, g \in \mathscr{K}(B, Y)$. Using this idea we can form a natural uniformity for $\mathscr{K}(B, Y)$, for $(Y, \mathscr{V})$ a uniform space, by taking all subsets of $\mathscr{K}(B, Y) \times \mathscr{K}(B, Y)$ of the form

$$
K_{V}=\{(f, g):(f(b), g(b)) \in V \text { for all } b \in B\},
$$

where $V$ runs over the elements of $\mathscr{V}$. It is easy to prove that the nonempty family $\mathscr{K}_{\mathscr{q}}=\left\{K_{V}: V \in \mathscr{Y}\right\}$ of subsets of $\mathscr{K}(B, Y) \times \mathscr{K}(B, Y)$ forms a base for a uniformity for $\mathscr{K}(B, Y)$. The topology induced on $\mathscr{K}(B, Y)$ is commonly called the topology of uniform convergence. We note that if $\mathscr{B}$ is a base for the uniformity $\mathscr{Y}$, then $\mathscr{K}_{\mathscr{B}}=$ $\left\{K_{V}: V \in \mathscr{B}\right\}$ generates the same uniformity for $\mathscr{K}(B, Y)$ as does $\mathscr{K}_{\Psi}$.

The following definition is due to Vala [32], although its contents arise in other works.

DEFINITION 2.2. A subset $\mathscr{H} \subset \mathscr{K}(B, Y)$ is said to have equal variation on $B$ if, for every $V \in \mathscr{V}$, there is a finite covering $B_{1}, \cdots$, $B_{n}$ of $B$ such that $a, b \in B_{i}, i=1, \cdots, n$, implies

$$
(f(a), f(b)) \in V
$$

for every $f \in \mathscr{H}$. We write this as $\left(f\left(B_{i}\right), f\left(B_{i}\right)\right) \subset V$ for $i=1, \cdots, n$ where $\left(f\left(B_{i}\right), f\left(B_{i}\right)\right)=\left\{(f(a), f(b)): a, b \in B_{i}\right\}$.

We could define equal variation for sets of arbitrary functions 
from $B$ into $Y$ although we have assumed them in $\mathscr{K}(B, Y)$ for the purposes of Definition 2.2. The following lemma, however, says that these functions must be precompact, as obviously a subset of a set having equal variation also has equal variation.

Lemma 2.1. An arbitrary function $f: B \rightarrow Y$ has equal variation if and only if $f \in \mathscr{K}(B, Y)$.

Proof. Let $f$ have equal variation. Then, if $V \in \mathscr{Y}$, there exists a finite cover $B_{1}, \cdots, B_{n}$ of $B$ such that $\left(f\left(B_{i}\right), f\left(B_{i}\right)\right) \subset V$ for $i=$ $1, \cdots, n$. Let $b_{i} \in B_{i}$, where we assume that any empty $B_{i}$ 's have been discarded. Thus $\left(f\left(b_{i}\right), f(b)\right) \in V$ for all $b \in B_{i}$. Hence $f(b) \in$ $V\left[f\left(b_{i}\right)\right]$, for all $b \in B_{i}$. As $\bigcup_{i=1}^{n} B_{i}=B$, we have that

$$
f(B) \subset \bigcup_{i=1}^{n} V\left[f\left(b_{i}\right)\right],
$$

which says that $f(B)$ is precompact.

Conversely, assume $f$ is precompact and let $V \in \mathscr{Y}$. Then, as $\mathscr{V}$ is a uniformity, there exists a symmetric $U \in \mathscr{V}$ (that is, $U=U^{-1}$ ) such that $U \circ U \subset V$. Since $f$ is precompact, there exists a finite subset $\left\{b_{1}, \cdots, b_{n}\right\} \subset B$ such that

$$
f(B) \subset \bigcup_{i=1}^{n} U\left[f\left(b_{i}\right)\right] \text {. }
$$

Hence $B \subset f^{-1}\left(\bigcup_{i=1}^{n} U\left[f\left(b_{i}\right)\right]\right)=\bigcup_{i=1}^{n} f^{-1}\left(U\left[f\left(b_{i}\right)\right]\right)$. Let $B_{i}=f^{-1}\left(U\left[f\left(b_{i}\right)\right]\right)$ and let $a, b \in B_{i}$. Then, obviously, $f(a), f(b) \in U\left[f\left(b_{i}\right)\right]$ and hence $\left(f\left(b_{i}\right), f(a)\right),\left(f\left(b_{i}\right), f(b)\right) \in U$. But $U=U^{-1}$ and hence $(f(a), f(b)) \in$ $U \circ U \subset V$ which implies that $\left(f\left(B_{i}\right), f\left(B_{i}\right)\right) \subset V$ for $i=1, \cdots, n$. Thus $f$ has equal variation.

The next Lemma is very useful as it allows the proof of Theorem 2.1 to flow more elegantly, being used for both directions of the proof. It is essentially a generalization of Lemma 2.1 and in fact generalizes a lemma of Goldberg [16, Lemma III.2.2, p. 84].

Lemma 2.2. Let $\mathscr{H}_{1}, \cdots, \mathscr{H}_{n}$ be subsets of $\mathscr{K}(B, Y)$ having equal variation. Then $\mathrm{U}_{i=1}^{n} \mathscr{H}_{i}$ has equal variation.

Proof. Let $V \in \mathscr{Y}$. By hypothesis, for each $k \in\{1, \cdots, n\}$ there exists a finite covering $\left\{B_{i_{k}}^{(k)}\right\}_{i_{k} \in I_{k}}$ of $B$ such that $\left(f\left(B_{i_{k}}^{(k)}\right), f\left(B_{i_{k}}^{(k)}\right)\right) \subset V$ for all $f \in \mathscr{C}_{k}, i_{k} \in I_{k}$. Obviously the sets

$$
\left\{B_{i_{1}}^{(1)} \cap \cdots \cap B_{i_{n}}^{(n)}\right\}_{\left(i_{1}, \cdots, i_{n}\right) \in I_{1} \times \cdots \times I_{n}}
$$

form a finite covering of $B$, although some may be empty. Let 
$f \in \bigcup_{i=1}^{n} \mathscr{K}_{i}$ and $a, b \in B_{i_{1}}^{(1)} \cap \cdots \cap B_{i_{n}}^{(n)}$. Then $f \in \mathscr{H}_{j}$ for some $j \in\{1, \cdots$, $n\}$ and $a, b \in B_{i_{j}}^{(j)}$. Accordingly, $(f(a), f(b)) \in V$. Hence $\bigcup_{i=1}^{n} \mathscr{H}_{i}$ has equal variation on $B$.

It is immediately obvious from Lemmas 2.1 and 2.2 that every finite subset of $\mathscr{K}(B, Y)$ has equal variation on $B$.

We shall now characterize the precompact subsets of $\mathscr{K}(B, Y)$ under the topology of uniform convergence. This is essentially an Ascoli-Arzelà theorem and the statement and proof follow the same lines as the metric space case of Vala [32]. The proof is included here as it is typical of so many of the arguments used in later theorems and it also uses Lemma 2.2 to give a slightly more efficient proof than that of Vala. As Vala [32] states, the theorem is not essentially a generalization of the Ascoli-Arzelà theorem but is only another form of it, adapted for certain applications. A result of Poppe [26] also comes out as a direct corollary.

Theorem 2.1. Let $\mathscr{H}$ be a subset of $\mathscr{K}(B, Y)$. Then $\mathscr{H}$ is precompact in $\mathscr{K}(B, Y)$, under the topology of uniform convergence, if and only if

(1) the set $\mathscr{H}(b)$ is precompact for every $b \in B$,

(2) $\mathscr{H}$ has equal variation on $B$.

Proof. Suppose $\mathscr{H}$ is precompact in $\mathscr{K}(B, Y)$. Let $b_{0} \in B$ and $V \in \mathscr{Y}$. As $\mathscr{C}$ is precompact, there exist functions $f_{1}, \cdots, f_{n} \in \mathscr{H}$ such that

$$
\mathscr{H} \subset \bigcup_{i=1}^{n} K_{V}\left[f_{i}\right]
$$

Thus, if $f \in \mathscr{H}$, there exists an $f_{i}$ such that $\left(f_{i}, f\right) \in K_{V}$ and hence, by definition, $\left(f_{i}(b), f(b)\right) \in V$ for all $b \in B$. In particular, $\left(f_{i}\left(b_{0}\right), f\left(b_{0}\right)\right) \in V$ or $f\left(b_{0}\right) \in V\left[f_{i}\left(b_{0}\right)\right]$ and hence

$$
\mathscr{H}\left(b_{0}\right) \subset \bigcup_{i=1}^{n} V\left[f_{i}\left(b_{0}\right)\right],
$$

proving that $\mathscr{H}\left(b_{0}\right)$ is precompact for all $b_{0} \in B$. Thus condition (1) is satisfied. We now prove condition (2), namely that $\mathscr{H}$ has equal variation on $B$. Let $V \in \mathscr{V}$. Then there exists a symmetric $U \in \mathscr{V}$ such that $U \circ U \circ U \subset V$. It is an immediate consequence of the definitions that $K_{U} \circ K_{U} \circ K_{U} \subset K_{V}$. Since $\mathscr{H}$ is precompact, there exist $f_{1}, \cdots, f_{n} \in \mathscr{H}$ such that

$$
\mathscr{H} \subset \bigcup_{i=1}^{n} K_{U}\left[f_{i}\right] .
$$


As each $f_{i}$ is precompact, the set $\left\{f_{1}, \cdots, f_{n}\right\}$ has equal variation on $B$ by Lemma 2.2. Thus there exists a finite cover $B_{1}, \cdots, B_{n}$ of $B$ such that $\left(f_{i}\left(B_{k}\right), f_{i}\left(B_{k}\right)\right) \subset U$ for all $k \in\{1, \cdots, m\}, i \in\{1, \cdots, n\}$. Let $f \in \mathscr{K}$ and $b_{1}, b_{2} \in B_{k}$ for some $k \in\{1, \cdots, m\}$. There exists an $i \in\{1, \cdots, n\}$ such that $f \in K_{U}\left[f_{i}\right]$ and hence $\left(f_{i}(b), f(b)\right) \in U$ for all $b \in B$. Now, as $b_{1}, b_{2} \in B_{k} \subset B$ and $U=U^{-1}$, we have that $\left(f\left(b_{1}\right), f_{2}\left(b_{1}\right)\right) \in U$, $\left(f_{i}\left(b_{1}\right), f_{i}\left(b_{2}\right)\right) \in U, \quad\left(f_{i}\left(b_{2}\right), f\left(b_{2}\right)\right) \in U$. Thus $\left(f\left(b_{1}\right), f\left(b_{2}\right)\right) \in U \circ U \circ U \subset V$ and it follows that $\left(f\left(B_{k}\right), f\left(B_{k}\right)\right) \subset V$ for all $k \in\{1, \cdots, m\}, f \in \mathscr{Z}$. We have now proved that $\mathscr{C}$ has equal variation on $B$ and (2) is satisfied.

Conversely, suppose now that (1) and (2) are satisfied. Let $K_{V} \in \mathscr{K}_{\%}$. Then there exists a symmetric $U \in \mathscr{Y}$ such that $U \circ U \circ U \subset$ $V$. As $\mathscr{H}$ has equal variation, there exists a finite cover $B_{1}, \cdots, B_{p}$ of $B$ such that $\left(f\left(B_{k}\right), f\left(B_{k}\right)\right) \subset U$ for all $k \in\{1, \cdots, p\}, f \in \mathscr{H}$. Assuming, without loss of generality, that each $B_{k}$ is nonempty, let $b_{k} \in B_{k}$ for $k \in\{1, \cdots, p\}$. By hypothesis (1), $\mathscr{\mathscr { C }}\left(b_{k}\right)$ is precompact in $Y$ for all $k \in\{1, \cdots, p\}$. We define the mappings $\Theta_{b}: \mathscr{H} \rightarrow Y$ by $\Theta_{b}(f)=f(b)$ for each $b \in B, f \in \mathscr{C}$. Then $\Theta_{b}(\mathscr{C})=\mathscr{X}(b)$ and hence $\Theta_{b}$ is a precompact mapping for all $b \in B$. By Lemma $2.2,\left\{\Theta_{b_{1}}, \cdots, \Theta_{b_{p}}\right\}$ has equal variation on $\mathscr{H}$. Hence there exists a finite cover $\mathscr{C}_{1}, \cdots$, $\mathscr{H}_{l}$ of $\mathscr{H}$ such that $\left(\Theta_{b_{k}}\left(\mathscr{K}_{j}\right), \Theta_{b_{k}}\left(\mathscr{C}_{j}\right)\right) \subset U$ for all $k \in\{1, \cdots, p\}$, $j \in\{1, \cdots, l\}$ or $\left(\mathscr{H}_{j}\left(b_{k}\right), \mathscr{H}_{j}\left(b_{k}\right)\right) \subset U$ for all $k \in\{1, \cdots, p\}, j \in\{1, \cdots, l\}$. Let $f_{j} \in \mathscr{K}_{j}$ for $j \in\{1, \cdots, l\}$, assuming that each $\mathscr{H}_{j}$ is nonempty. Then we claim that

$$
\mathscr{H} \subset \bigcup_{j=1}^{l} K_{V}\left[f_{j}\right]
$$

proving $\mathscr{H}$ is precompact. For, if $f \in \mathscr{H}$, then $f \in \mathscr{H}_{j}$ for some $j \in\{1, \cdots, l\}$. We prove that $f \in K_{\square}\left[f_{j}\right]$ or $\left(f_{j}(b), f(b)\right) \in V$ for all $b \in B$. If $b \in B, b \in B_{k}$ for some $k \in\{1, \cdots, p\}$. But, as $f \in \mathscr{H}_{j},\left(f_{j}\left(b_{k}\right), f\left(b_{k}\right)\right) \in U$ by the above work and, as $\mathscr{C}$ has equal variation on $B$ and by construction of $B_{k}$ 's $\left(f_{j}\left(b_{k}\right), f_{j}(b)\right) \in U$ and $\left(f\left(b_{k}\right), f(b)\right) \in U$. As $U$ is symmetric, $\left(f_{j}(b), f(b)\right) \in U \circ U \circ U \subset V$. This completes the proof.

Let $\mathscr{S}$ be a collection of subsets of $B$ which is directed under set-theoretic inclusion $\subset$.

Definition 2.3. A mapping $f: B \rightarrow Y$ is said to be $\mathscr{S}$-precompact (S-compact) if $f(A)$ is precompact (relatively compact) in $Y$ for all $A \in \mathscr{S}_{\text {. Let }} \mathscr{K}_{\mathscr{S}}(B, Y)$ denote the set of all $\mathscr{S}$-precompact mappings from $B$ into $Y$.

The subsets $K_{A, V}=\{(f, g):(f(a), g(a)) \in V$ for all $a \in A\}$ of $\mathscr{K}_{S}(B, Y) \times \mathscr{K}_{\mathcal{S}}(B, Y)$ form a base for a uniformity for $\mathscr{K}_{S}(B, Y)$. The topology induced on $\mathscr{K}_{S}(B, Y)$ is commonly called the topology 
of uniform convergence on elements of $\mathscr{S}$.

The following corollary to Theorem 2.1 is a result due to Poppe [26].

Corollary. A subset $\mathscr{H} \subset \mathscr{K}_{\mathscr{S}}(B, Y)$ is precompact under the topology defined above if and only if

(1) $\mathscr{H}(x)$ is precompact for all $x \in \mathbf{U}_{A \in \mathscr{S}} A$,

(2) $\left.\mathscr{H}\right|_{A}$ has equal variation on $A$ for all $A \in \mathscr{S}$.

Equivalently, $\mathscr{H}$ is precompact in $\mathscr{K}_{\mathscr{S}}(B, Y)$ if and only if $\left.\mathscr{H}\right|_{A}$, considered as a subset of $\mathscr{K}(A, Y)$, is precompact for all $A \in \mathscr{S}$.

Proof. Let $\mathscr{H}$ be precompact in $\mathscr{K}_{\mathscr{S}}(B, Y)$ and let $A \in \mathscr{S}$. The mapping

$$
\mathscr{R}_{A}: \mathscr{K}_{\mathscr{Y}}(B, Y) \longrightarrow \mathscr{K}(A, Y)
$$

defined by $\mathscr{R}_{A}(f)=\left.f\right|_{A}$ is easily shown to be uniformly continuous. Since a uniformly continuous image of a precompact set is precompact, $\mathscr{R}_{A}(\mathscr{H})=\left.\mathscr{H}\right|_{A}$ is a precompact subset of $\mathscr{K}(A, Y)$. Hence, by Theorem 2.1, $\left.\mathscr{C}\right|_{A}$ has equal variation on $A$ for all $A \in \mathscr{S}$, implying that (2) is satisfied. Also, if $x \in \bigcup_{A \in \mathscr{S}} A$, then $x \in A$ for some $A \in \mathscr{S}$ and again, as $\left.\mathscr{H}\right|_{A}$ is precompact in $\mathscr{K}(A, Y), \mathscr{H}(x)$ is precompact in $Y$ by Theorem 2.1. Thus conditions (1) and (2) are satisfied.

Conversely, suppose conditions (1) and (2) hold. Then, by Theorem 2.1, $\left.\mathscr{C}\right|_{A}$ is precompact in $\mathscr{K}(A, Y)$ for every $A \in \mathscr{S}$. We must prove that $\mathscr{C}$ is a precompact subset of $\mathscr{K}_{\mathcal{U}}(B, Y)$. Let $K_{A, V}$ be a basic member of the uniformity for $\mathscr{K}_{S}(B, Y)$, where $A \in \mathscr{S}, V \in \mathscr{Y}$. Now $K(A)_{V}=\{(f, g): f, g \in \mathscr{K}(A, Y),(f(a), g(a)) \in V$ for all $a \in A\}$ is a member of the uniformity for $\mathscr{K}(A, Y)$. As $\left.\mathscr{H}\right|_{A}$ is precompact in $\mathscr{K}(A, Y)$, there exist $f_{1}, \cdots, f_{n} \in \mathscr{C}$ such that

$$
\left.\mathscr{H}\right|_{A} \subset \bigcup_{i=1}^{n} K(A)_{V}\left[\left.f_{i}\right|_{A}\right]
$$

If $f \in \mathscr{H}$, there exists an $f_{i}, i \in\{1, \cdots, n\}$, such that $\left.f\right|_{A} \in K(A)_{V}\left[\left.f_{i}\right|_{A}\right]$. Hence $\left(f_{i}(a), f(a)\right) \in V$ for all $a \in A$, which means $\left(f_{i}, f\right) \in K_{A, V}$. Thus

$$
\mathscr{L} \subset \bigcup_{i=1}^{n} K_{A, r}\left[f_{i}\right],
$$

which implies that $\mathscr{H}$ is precompact in $\mathscr{K}_{S}(B, Y)$. This completes the proof.

The following definition is well known [20, Problem 7.G, p. 239].

Definition 2.4. A family $\mathscr{H}$ of functions from a uniform space $(X, \mathscr{U})$ into a uniform space $(Y, \mathscr{V})$ is uniformly equicontinuous iff 
for each $V \in \mathscr{V}$ there is a $U \in \mathscr{U}$ such that $(f(x), f(y)) \in V$ for all $f \in \mathscr{H},(x, y) \in U$.

For the remainder of this section, we let $(B, \mathscr{C})$ be a precompact uniform space and $(Y, \mathscr{V})$ a uniform space. We have

Proposition 2.1. Let $\mathscr{H}$ be a uniformly equicontinuous set of mappings from $B$ into $Y$. Then $\mathscr{H}$ has equal variation on $B$.

Proof. Let $V \in \mathscr{Y}$ and let $W \in \mathscr{Y}$ such that $W \circ W \subset V$ and $W=W^{-1}$. As $\mathscr{H}$ is uniformly equicontinuous, there exists a $U \in \mathscr{U}$ such that $(f(x), f(y)) \in W$ for all $f \in \mathscr{H},(x, y) \in U$. Now, as $B$ is precompact, there exists a finite subset $\left\{b_{1}, \cdots, b_{n}\right\} \subset B$ such that

$$
B=\bigcup_{i=1}^{n} U\left[b_{i}\right] \text {. }
$$

Thus $U\left[b_{i}\right]$ is a finite cover of $B$ and we claim that

$$
\left(f\left(U\left[b_{i}\right]\right), f\left(U\left[b_{i}\right]\right)\right) \subset V \quad f \in \mathscr{H}, \quad i=1, \cdots, n .
$$

For, if $a, b \in U\left[b_{i}\right]$, then $\left(b_{i}, a\right) \in U,\left(b_{i}, b\right) \in U$. Hence $\left(f\left(b_{\imath}\right), f(a)\right) \in W$ and $\left(f\left(b_{i}\right), f(b)\right) \in W$ for all $f \in \mathscr{H}$. But this means that $(f(a), f(b)) \in$ $W \circ W \subset V$, as $W=W^{-1}$. Accordingly, $\mathscr{H}$ has equal variation on $B$.

Setting $\mathscr{H}$ to be a singleton set in Definition 2.4 gives us the definition of a uniformly continuous function.

COROLLARY. Let $\mathscr{H}$ be a uniformly equicontinuous set of mappings from $B$ into $Y$. Then, if $\mathscr{H}(b)$ is precompact in $Y$ for all $b \in B$, $\mathscr{H}$ is a precompact subset of $\mathscr{K}(B, Y)$ with the topology defined previously.

Proof. Firstly, $\mathscr{H} \subset \mathscr{K}(B, Y)$ by the fact that each $f \in \mathscr{H}$ is uniformly continuous and $B$ is precompact. By Proposition 2.1, $\mathscr{C}$ has equal variation. Hence conditions (1) and (2) of Theorem 2.1 are satisfied by the added hypothesis. This proves that $\mathscr{H}$ is precompact.

3. Precompact sets of semi-precompact operators. Throughout this section we let $X$ and $Y$ be topological vector spaces over the complex numbers $C$; these are indeed uniform spaces and hence the results of $\$ 2$ carry over. We interpret the definition of equal variation in this case as: A subset $\mathscr{H} \subset \mathscr{K}(B, Y)$ (where $B$ is an arbitrary set) has equal variation on $B$ if, for any neighborhood $V$ of zero in $Y$, there exists a finite cover $B_{1}, \cdots, B_{n}$ of $B$ such that

$$
f\left(B_{i}\right)-f\left(B_{i}\right)=\left\{f(a)-f(b): a, b \in B_{i}\right\} \subset V
$$

for all $f \in \mathscr{H}, i=1, \cdots, n$. 
We use the notation and results of Treves [31, Chapter 32] in what follows. Let $\mathscr{S}$ be a family of bounded subsets of $X$ satisfying the conditions:

$\left(\mathscr{S}_{1}\right)$ if $A, B \in \mathscr{S}$, then there is a $C \in \mathscr{S}$ such that $A \cup B \subset C$,

$\left(\mathscr{S}_{2}\right)$ if $\lambda \in C$ and $A \in \mathscr{S}$, then there is a $B \in \mathscr{S}$ such that $\lambda A \subset B$. The $\mathscr{S}$-topology on $\mathscr{L}[X, Y]$ is called the topology of uniform convergence on elements of $\mathscr{S}$ and when carrying the $\mathscr{S}$-topology we write it as $\mathscr{L}_{S}[X, Y] . \mathscr{L}_{S}[X, Y]$ is a topological vector space [31, p. 336] and when $\mathscr{S}$ is a fundamental system of bounded sets in $X$ (that is, any bounded subset of $X$ is contained in some member of $\mathscr{S})$ we denote $\mathscr{L}_{S}[X, Y]$ by $\mathscr{L}_{5}[X, Y]$.

We let $\mathscr{K}_{S}[X, Y]$ denote the set of all continuous $\mathscr{S}$-precompact linear operators from $X$ into $Y$ with the topology induced by $\mathscr{L}_{S}[X, Y]$. Obviously $\mathscr{K}_{S}[X, Y]$ is a linear subspace of $\mathscr{L}_{S}[X, Y]$ by $[28$, Lemma 3, p. 49] and it is easy to prove that the topology on $\mathscr{K}_{S}[X, Y]$ coincides with the induced topology on $\mathscr{K}_{\mathscr{S}}[X, Y]$ considered as a subset of $\mathscr{K}_{S}(X, Y)$.

The following is a generalization of Vala's characterization of a precompact set of precompact operators from a normed linear space $X$ into a normed linear space $Y[32, \S 6$, p. 6$]$.

THEOREM 3.1. Let $X$ and $Y$ be topological vector spaces and $\mathscr{S}$ some collection of bounded subsets of $X$ satisfying conditions $\left(\mathscr{S}_{1}\right)$ and $\left(\mathscr{S}_{2}\right)$. Then a subset $\mathscr{H} \subset \mathscr{K}_{s}[X, Y]$ is precompact if and only if

(1) $\mathscr{H}(x)$ is precompact for all $x \in \bigcup_{B \in \mathscr{S}} B$,

(2) $\left.\mathscr{H}\right|_{B}$ has equal variation on $B$ for all $B \in \mathscr{S}$.

Equivalently, $\mathscr{C}$ is precompact in $\mathscr{K}_{s}[X, Y]$ if and only if $\left.\mathscr{H}\right|_{B}$, considered as a subset of $\mathscr{K}(B, Y)$, is precompact for every $B \in \mathscr{S}$.

Proof. This is obvious from the Corollary to Theorem 2.1, since a subset $\mathscr{H} \subset \mathscr{K}_{S}[X, Y]$ is precompact if and only if $\mathscr{H}$ is precompact when considered as a subset of $\mathscr{K}_{S}(X, Y)$.

This general theorem gives, as a trivial consequence, a characterization of the precompact subsets of $\mathscr{K}_{\varpi}[X, Y]$, the set of all semi-precompact continuous linear operators with the topology of uniform convergence on bounded subsets of $X$.

4. A generalization of Schauder's theorem. Let $W, X, Y$, and $Z$ be four locally convex Hausdorff spaces. Given three continuous linear operators $R \in \mathscr{L}[Y, Z], T \in \mathscr{L}[X, Y]$, and $S \in \mathscr{L}[W, X]$, we can form the composition operator $R T S$ : 


$$
W \stackrel{S}{\longrightarrow} X \stackrel{T}{\longrightarrow} Y \stackrel{R}{\longrightarrow} Z,
$$

which is a continuous linear operator in $\mathscr{L}[W, Z]$. Keeping $R$ and $S$ fixed and allowing $T$ to vary over $\mathscr{L}[X, Y]$, we have a mapping

$$
\Phi: \mathscr{L}[X, Y] \longrightarrow \mathscr{L}[W, Z]
$$

defined by $\Phi(T)=R T S$.

Lemma 4.1. Let $W, X, Y$, and $Z$ be locally convex Hausdorff spaces and let $R \in \mathscr{L}[Y, Z]$ and $S \in \mathscr{L}[W, X]$. Then the mapping $\Phi: \mathscr{L}_{5}[X, Y] \rightarrow \mathscr{L}_{b}[W, Z]$, defined by $\Phi(T)=R T S$, is a continuous linear operator.

Proof. It is obvious that $\Phi$ is linear. Let $q$ be a continuous seminorm on $Z, \varepsilon>0$ and $B$ a bounded subset of $W$. Then $\mathscr{V}(B ; q ; \varepsilon)$ is an arbitrary neighborhood of zero in $\mathscr{L}_{b}[W, Z]$, where

$$
\mathscr{Y}(B ; q ; \varepsilon)=\left\{P \in \mathscr{L}[W, Z]: q_{B}(P) \leqq \varepsilon\right\}
$$

$\left(q_{B}(P)=\sup _{b \in B} q(P(b))\right)$. As $S$ is continuous, $S(B)$ is a bounded subset of $X$ [28, Proposition 1, p. 45]. Also, as $q$ is a continuous seminorm and $R$ is a continuous linear mapping, $q \circ R$ is a continuous seminorm on $Y$. The set

$$
\mathscr{U}(S(B) ; q \circ R ; \varepsilon)=\left\{T \in \mathscr{C}[X, Y]:(q \circ R)_{S(B)}(T) \leqq \varepsilon\right\}
$$

is a neighborhood of zero in $\mathscr{L}_{5}[X, Y]$. Now

$$
\Phi(\mathscr{Z}(S(B) ; q \circ R ; \varepsilon)) \subset \mathscr{Y}(B ; q ; \varepsilon) .
$$

In fact, if $T \in \mathscr{C}(S(B) ; q \circ R ; \varepsilon)$, then $(q \circ R)_{S(B)}(T) \leqq \varepsilon$. However, $(q \circ R)_{S(B)}(T)=\sup _{b \in B}(q \circ R)(T S(b))=\sup _{b \in B} q(R T S(b))=\sup _{b \in B} q(\Phi(T)(b))=$ $q_{B}(\Phi(T))$, which implies $q_{B}(\Phi(T)) \leqq \varepsilon$. Thus $\Phi(T) \in \mathscr{C}(B ; q ; \varepsilon)$. Hence $\Phi$ is continuous and the proof is complete.

The mapping $\Phi$ depends on the mappings $R$ and $S$, so we would expect their qualities to be somehow linked with those of $\Phi$. This is shown, in one respect, in Theorem 4.1 below.

Firstly, we give a property of infrabarrelled spaces. A locally convex space $X$ is infrabarrelled [18, Definition 2, p. 217] if every bornivorous (absorbs bounded sets) barrel in $X$ is a neighborhood of zero, where a barrel is an absorbing, balanced, convex and closed subset of $X$. Let $\beta\left(X^{\prime}, X\right)$ be the strong topology on $X^{\prime}$, the continuous dual of $X$. This means that $X^{\prime}$ with the topology $\beta\left(X^{\prime}, X\right)$ is just the space $\mathscr{L}_{5}[X, C]$. By [21, Theorem 20.3, p. 191], $X$ is infrabarrelled if and only if every $\beta\left(X^{\prime}, X\right)$-bounded set in $X^{\prime}$ is 
equicontinuous. Another equivalent characterization is given in [21, Theorem 20.3, p. 191] and states that $X$ is infrabarrelled if and only if the evaluation map $I: X \rightarrow X^{\prime \prime}$, given by $I(x)(f)=f(x)$, is continuous, where $X^{\prime \prime}$ is the continuous dual of $X^{\prime}$ when it has the $\beta\left(X^{\prime}, X\right)$ topology and $X^{\prime \prime}$ has the $\beta\left(X^{\prime \prime}, X^{\prime}\right)$ topology. The following is a slightly stronger characterization and is included here because of the lack of a reference although [29, Theorem 4.2, p. 83] is a close result for $X$ barrelled.

Lemma 4.2. Let $X$ and $Y$ be locally convex spaces. Then $X$ is infrabarrelled if and only if every bounded subset of $\mathscr{L}_{b}[X, Y]$ is equicontinuous.

Proof. By [29, Proposition 3.3, p. 81], a subset $\mathscr{B} \subset \mathscr{L}_{5}[X, Y]$ is bounded if and only if, for every neighborhood $V$ of zero in $Y$, $\bigcap_{T \in \mathscr{B}} T^{-1}(V)$ absorbs every bounded set in $X$, that is $\bigcap_{T \in \mathscr{B}} T^{-1}(V)$ is bornivorous in $X$. (It is easily deduced from this that every equicontinuous set is bounded.)

Suppose $\mathscr{B} \subset \mathscr{L}_{5}[X, Y]$ is bounded and $X$ is infrabarrelled. Let $V$ be a convex, balanced, closed neighborhood of zero in $Y$. Then $\bigcap_{T \in \mathscr{G}} T^{-1}(V)$ is a bornivorous barrel in $X$, as $\mathscr{B}$ is bounded and each $T \in \mathscr{B}$ is continuous and linear. Thus it is a neighborhood of zero, as $X$ is infrabarrelled. Hence $\mathscr{B}$ is equicontinuous by [29, Proposition 4.1, p. 83] and the fact that $V$ is an arbitrary basic neighborhood of zero (as $Y$ is locally convex).

The converse is obvious by setting $Y=C$ the complex numbers and using the characterization mentioned earlier.

The following result is used in Theorem 4.1 and is a direct consequence of [29, Proposition 3.3, p. 81].

Lemma 4.3. Let $X$ and $Y$ be locally convex spaces and $\mathscr{B} \subset$ $\mathscr{L}_{5}[X, Y]$ be bounded. Then $\mathscr{B}(M)=\bigcup_{T \in \mathscr{B}} T(M)$ is bounded in $Y$ for all bounded subsets $M$ of $X$. In particular, $\mathscr{B}(x)$ is bounded in $Y$ for all $x \in X$.

Now we have the locally convex case of the generalization of Schauder's theorem proved by Vala [32, Theorem 3, p. 6] for normd spaces. A condition such as $X$ infrabarrelled is to be expected in the theorem, for, if $Y=Z=C$ and $R$ is the identity map, the known generalization of Schauder's theorem to locally convex spaces [21, Problem 21D, p. 208] falls out as a corollary.

TheOREM 4.1. Let $W, X, Y$, and $Z$ be locally convex Hausdorff spaces and in addition assume $X$ is infrabarrelled. Let $R \in \mathscr{L}[Y, Z]$, 
$S \in \mathscr{L}[W, X]$ both be nonzero. Then the mapping

$$
\Phi: \mathscr{L}_{b}[X, Y] \longrightarrow \mathscr{L}_{5}[W, Z],
$$

given by $\Phi(T)=R T S$, is a semi-precompact continuous linear operator if and only if both $R$ and $S$ are semi-precompact.

Proof. Let $R$ and $S$ be nonzero and semi-precompact. Suppose $\mathscr{B} \subset \mathscr{L}_{5}[X, Y]$ is bounded. Set

$$
\mathscr{H}=\Phi(\mathscr{B})=\{H \in \mathscr{L}[W, Z]: H=R T S \text { for some } T \in \mathscr{B}\} .
$$

We must show that $\Phi(\mathscr{B})=\mathscr{H}$ is a precompact subset of $\mathscr{L}_{b}[W, Z]$. This is equivalent to proving that $\left.\mathscr{H}\right|_{M_{W}}$ has equal variation on $M_{W}$ for all bounded $M_{W} \subset W$ and $\mathscr{C}(w)$ is precompact for each $w \in W$ by Theorem 3.1, as obviously $\mathscr{H}$ consists of semi-precompact operators.

Firstly, let $w \in W$, which implies that $S(w) \in X$ and hence $\mathscr{B} S(w)$ is bounded in $Y$ by Lemma 4.3. By assumption $R$ is semi-precompact and thus $R \mathscr{B} S(w)=\mathscr{H}(w)$ is precompact in $Z$, fulfilling condition (1) of Theorem 3.1.

Now let $M_{W}$ be bounded in $W$ and let $V_{Z}$ be a neighborhood of zero in $Z$. As $R$ is continuous, there exists a neighborhood $V_{Y}$ of zero in $Y$ such that $R\left(V_{Y}\right) \subset V_{Z} . \mathscr{P}$ is bounded in $\mathscr{L}_{b}[X, Y]$ and hence, by Lemma $4.2, \mathscr{B}$ is equicontinuous as $X$ is infrabarrelled. This implies that there exists a neighborhood $V_{X}$ of zero in $X$ such that $\mathscr{B}\left(V_{X}\right) \subset V_{Y} . \quad S$ is semi-precompact and obviously $\{S\}$ is precompact in $\mathscr{L}_{5}[W, X]$ which implies, by Theorem 3.1, that $\left.S\right|_{M_{W}}$ has equal variation. Let $M_{1}, \cdots, M_{n}$ be a finite cover of $M_{W}$ such that $S\left(M_{i}\right)-S\left(M_{i}\right) \subset V_{X}$ for $i=1, \cdots, n$. Then we have $R \mathscr{B}\left(S\left(M_{i}\right)-\right.$ $\left.S\left(M_{i}\right)\right) \subset R \mathscr{B}\left(V_{X}\right) \subset R\left(V_{Y}\right) \subset V_{Z}$ for $i=1, \cdots, n$. Hence $R T\left(S\left(M_{i}\right)-\right.$ $\left.S\left(M_{i}\right)\right) \subset V_{Z}$ for $i=1, \cdots, n, T \in \mathscr{B}$. As $R T$ is linear for all $T \in \mathscr{B}$, we have that $R T S\left(M_{i}\right)-R T S\left(M_{i}\right) \subset V_{z}$ for $i=1, \cdots, n, T \in \mathscr{B}$ or equivalently $H\left(M_{i}\right)-H\left(M_{i}\right) \subset V_{Z}$ for $i=1, \cdots, n, H \in \mathscr{H}$. Thus $\left.\mathscr{H}\right|_{M_{W}}$ has equal variation on $M_{W}$ and we have proved that $\Phi(\mathscr{B})$ is precompact in $\mathscr{L}_{b}[W, Z]$. Consequently, $\Phi$ is semi-precompact and we know that it is continuous by Lemma 4.1.

Conversely, suppose $\Phi: \mathscr{L}_{b}[X, Y] \rightarrow \mathscr{L}_{5}[W, Z]$ is semi-precompact. We prove that $R$ and $S$ are semi-precompact, assuming of course that $R$ and $S$ are nonzero.

Firstly, $R$ is proved semi-precompact. Let $M_{Y}$ be a bounded subset of $Y$. We must prove that $R\left(M_{Y}\right)$ is precompact in $Z$. As $S$ is nonzero, there exists a $w_{0} \in W$ such that $S\left(w_{0}\right) \neq 0$. As $X$ is locally convex Hausdorff, there exists a continuous linear functional $f \in X^{\prime}$ such that $f\left(S\left(w_{0}\right)\right)=1$ [29, Theorem 4.2, Corollary 1, p. 49]. For each $y \in M_{Y}$ we define 


$$
K_{y}(x)=f(x) y \text {. }
$$

Then each operator $K_{y}: X \rightarrow Y$ is obviously a continuous linear onedimensional operator. Let

$$
\mathscr{K}=\left\{K_{y}: y \in M_{Y}\right\} \text {. }
$$

We show that $\mathscr{K}$ is bounded in $\mathscr{L}_{\mathrm{b}}[X, Y]$, which is equivalent to proving $\mathscr{C}$ equicontinuous, as $X$ is infrabarrelled (Lemma 4.2). Let $V_{Y}$ be a basic neighborhood of zero in $Y$. Then there exist a continuous seminorm $q$ on $Y$ such that

$$
V_{Y}=\{y \in Y: q(y) \leqq 1\} \text {. }
$$

$M_{Y}$ bounded in $Y$ implies there exists an $\alpha>0$ such that $\alpha M_{Y} \subset V_{Y}$. As $f$ is continuous, there exists a neighborhood $V_{X}$ of zero in $X$ such that $\left|f\left(V_{X}\right)\right| \leqq 1$ (i.e., $|f(x)| \leqq 1$ for all $\left.x \in V_{X}\right)$. Now $\mathscr{K}\left(\alpha V_{X}\right) \subset$ $V_{Y}$. In fact, let $K_{y} \in \mathscr{K}$ and $x \in V_{X}$. Then $q\left(K_{y}(\alpha x)\right)=q(f(\alpha x) y)=$ $q(f(x) \alpha y)=|f(x)| q(\alpha y) \leqq q(\alpha y)$, since $\left|f\left(V_{X}\right)\right| \leqq 1$. But, since $\alpha M_{Y} \subset$ $V_{Y}, q(\alpha y) \leqq 1$ and hence $q\left(K_{y}(\alpha x)\right) \leqq 1$. As $\alpha V_{X}$ is a neighborhood of zero in $X$, we have that $\mathscr{K}$ is equicontinuous and hence bounded in $\mathscr{L}_{b}[X, Y]$. But $\Phi$ is semi-precompact, implying that $\Phi(\mathscr{K})$ is precomact in $\mathscr{L}_{b}[W, Z]$. Theorem 3.1 then implies that $\Phi(\mathscr{K})(w)$ is precompact in $Z$ for all $w \in W$. Now $\Phi(\mathscr{K})\left(w_{0}\right)=\left\{R K_{y} S\left(w_{0}\right): y \in M_{Y}\right\}=$ $\left\{R\left(f\left(S\left(w_{0}\right)\right) y\right): y \in M_{Y}\right\}=\left\{R(y): y \in M_{Y}\right\}$, since $f\left(S\left(w_{0}\right)\right)=1$. Thus $\Phi(\mathscr{K})\left(w_{0}\right)=R\left(M_{Y}\right)$ and hence $R\left(M_{Y}\right)$ is precompact. As $M_{Y}$ is an arbitrary bounded subset of $Y, R$ is a semi-precompact operator.

Now we show that $S$ is semi-precompact. As $R \neq 0$, there exists a $y_{0} \in Y$ such that $R\left(y_{0}\right) \neq 0$. As $Y$ is locally convex and Hausdorff, there exists a continuous seminorm $q$ on $Y$ such that $q\left(y_{0}\right)>0$. We can assume without loss of generality that $q\left(y_{0}\right)=1$, by taking some positive multiple of $y_{0}$ or $q$ if necessary. Also, as $Z$ is locally convex and Hausdorff, there exists a continuous seminorm $r$ on $Z$ such that $r\left(R\left(y_{0}\right)\right)>0$. If $p$ is an arbitrary continuous seminorm on $X$, then

$$
V_{X}=\{x \in X: p(x) \leqq 1\}
$$

is an arbitrary basic neighborhood of zero in $X$ (as any positive multiple of a continuous seminorm is a continuous seminorm). Define

$$
V_{Z}=\left\{z \in Z: r(z) \leqq r\left(R\left(y_{0}\right)\right)\right\},
$$

which is a neighborhood of zero in $Z$, as $r\left(R\left(y_{0}\right)\right)>0$. We shall prove the existence of a bounded set $\mathscr{K} \subset \mathscr{L}_{b}[X, Y]$ such that

$$
\bigcap_{K \in \mathscr{F}} K^{-1} R^{-1}\left(V_{Z}\right) \subset V_{X}
$$


as well as having other special properties. As a consequence of the Hahn-Banach theorem [24, Proposition 8, p. 191] and because $p$ is continuous, for each $x_{0}$ fixed in $X$, there is a continuous linear functional $f \in X^{\prime}$ such that

$$
|f(x)| \leqq p(x) \text { for all } x \in X
$$

and

$$
f\left(x_{0}\right)=p\left(x_{0}\right)
$$

Define

$$
F_{p}=\left\{f \in X^{\prime}:|f(x)| \leqq p(x) \text { for all } x \in X\right\} \text {, }
$$

which is nonempty by the theorem mentioned above. Let

$$
\mathscr{K}=\left\{K_{f}: f \in F_{p}\right\},
$$

where $K_{f}(x)=f(x) y_{0}$. Obviously each $K_{f}$ is a continuous linear onedimensional mapping from $X$ into $Y$ and hence $\mathscr{C} \subset \mathscr{L}_{b}[X, Y]$. We claim that

$$
\bigcap_{K \in \mathscr{C}} K^{-1} R^{-1}\left(V_{z}\right) \subset V_{X} .
$$

Let $x \in \bigcap_{K \in \mathscr{K}} K^{-1} R^{-1}\left(V_{Z}\right)$. Then $x \in K^{-1} R^{-1}\left(V_{Z}\right)$ for all $K \in \mathscr{K}$, which implies that $R K(x) \in V_{Z}$ for all $K \in \mathscr{K}$. Hence

$$
r(R K(x)) \leqq r\left(R\left(y_{0}\right)\right) \quad \text { for all } K \in \mathscr{K},
$$

which means, by definition of $\mathscr{K}$,

$$
r\left(f(x) R\left(y_{0}\right)\right) \leqq r\left(R\left(y_{0}\right)\right) \quad \text { for all } f \in F_{p} .
$$

Hence $|f(x)| r\left(R\left(y_{0}\right)\right) \leqq r\left(R\left(y_{0}\right)\right)$ or $|f(x)| \leqq 1$ (as $\left.r\left(R\left(y_{0}\right)\right)>0\right)$ for all $f \in F_{p}$. Using [24, Proposition 8, p. 191], there exists an $f \in F_{p}$ such that $f(x)=p(x)$ and hence $p(x) \leqq 1$. Hence $x \in V_{X}$.

$\mathscr{K}$ is in fact an equicontinuous subset of $\mathscr{L}[X, Y]$. By definition of $V_{X}$ and $F_{p}$, we have

$$
\left|F_{p}\left(V_{X}\right)\right|=\sup _{f \in F_{p}} \sup _{x \in V_{X}}|f(x)| \leqq 1 .
$$

Let $V_{Y}=\{y \in Y: q(y) \leqq 1\}$ be an .arbitrary basic neighborhood of zero in $Y$, where $q$ is some continuous seminorm. If $q\left(y_{0}\right)=0$, then $q\left(K_{f}(x)\right)=q\left(f(x) y_{0}\right)=|f(x)| q\left(y_{0}\right)=0$ for all $f \in F_{p}, x \in X$ and hence

$$
\mathscr{K}(X) \subset V_{Y} ;
$$

if $q\left(y_{0}\right)>0$, we claim that 


$$
\mathscr{K}\left(\frac{1}{q\left(y_{0}\right)} V_{X}\right) \subset V_{Y},
$$

since, if $x \in V_{X}, \quad K_{f} \in \mathscr{K}$, then $q\left(K_{f}\left(1 / q\left(y_{0}\right) x\right)\right)=q\left(f\left(x / q\left(y_{0}\right)\right) y_{0}\right)=$ $1 / q\left(y_{0}\right) q\left(f(x) y_{0}\right)=|f(x)| q\left(y_{0}\right) / q\left(y_{0}\right) \leqq 1$. Hence $\mathscr{K}$ is equicontinuous and, in particular, bounded in $\mathscr{L}_{5}[X, Y]$.

Let $M_{W}$ be an arbitrary bounded subset of $W$. We must prove that $S\left(M_{W}\right)$ is a precompact subset of $X$. Let $V_{X}$ be an arbitrary basic neighborhood of zero in $X$. We can construct $\mathscr{K}$ from the associated continuous seminorm $p$ of $V_{X}$ as we have done above. $\mathscr{K}$ is bounded in $\mathscr{L}_{b}[X, Y]$ and, as $\Phi$ is semi-precompact, $\Phi(\mathscr{K})$ is precompact in $\mathscr{L}_{b}[W, Z]$. By Theorem 3.1, $\left.\Phi(\mathscr{K})\right|_{M_{W}}$ has equal variation on $M_{W}$. Hence there exists a finite cover $M_{1}, \cdots, M_{n}$ of $M_{W}$ such that

$$
\Phi(K)\left(M_{i}\right)-\Phi(K)\left(M_{i}\right) \subset V_{Z} \quad i=1, \cdots, n, K \in \mathscr{K},
$$

where $V_{Z}$ is defined as before. Thus

$$
\begin{array}{ll}
R K S\left(M_{i}\right)-R K S\left(M_{i}\right) \subset V_{Z} & i=1, \cdots, n, K \in \mathscr{K} \\
\Longrightarrow S\left(M_{i}\right)-S\left(M_{i}\right) \subset K^{-1} R^{-1}\left(V_{Z}\right) & i=1, \cdots, n, K \in \mathscr{K} \\
\Longrightarrow S\left(M_{i}\right)-S\left(M_{i}\right) \subset \bigcap_{K \in \mathscr{K}} K^{-1} R^{-1}\left(V_{Z}\right) & i=1, \cdots, n .
\end{array}
$$

By construction of $\mathscr{K}$ and $V_{Z}, \bigcap_{K \in \mathscr{K}} K^{-1} R^{-1}\left(V_{Z}\right) \subset V_{X}$ and hence

$$
S\left(M_{i}\right)-S\left(M_{i}\right) \subset V_{X} \quad i=1, \cdots, n .
$$

Thus $S\left(M_{W}\right)$ is precompact in $X$ (as $S \in \mathscr{K}\left(M_{W}, X\right)$ by Lemma 2.1), which implies that $S$ is a semi-precompact operator. This completes the proof.

The following corollary is Schauder's theorem (first proved for Banach spaces in [30]) for locally convex spaces as it appears in [21, Problem 21D, p. 208].

Corollary. Let $W$ and $X$ be locally convex Hausdorff spaces with $X$ infrabarrelled. Then a continuous linear operator $S: W \rightarrow$ $X$ is semi-precompact if and only if the adjoint operator $S^{\prime}: X^{\prime} \rightarrow$ $W^{\prime}$ is semi-precompact, where $X^{\prime}, W^{\prime}$ have their $\beta\left(X^{\prime}, X\right), \beta\left(W^{\prime}, W\right)$ topologies respectively.

Proof. Let $Y=Z=C$ in Theorem 4.1 and let $R$ be the identity map, which is obviously continuous, linear and semi-precompact. Then we have

$$
W \stackrel{S}{\longrightarrow} X \stackrel{f}{\longrightarrow} C \stackrel{I}{\longrightarrow} C
$$

and $\Phi: X^{\prime} \rightarrow W^{\prime}$ defined by $\Phi(f)=I f S=f S$, where $X^{\prime}=\mathscr{L}[X, \boldsymbol{C}]$ 
and $W^{\prime}=\mathscr{L}[W, \boldsymbol{C}]$, and $X^{\prime}$ (respectively $W^{\prime}$ ) with its strong topo$\log \mathrm{y} \beta\left(X^{\prime}, X\right)$ (respectively $\beta\left(W^{\prime}, W\right)$ ) is just $\mathscr{L}_{5}[X, C]$ (respectively $\left.\mathscr{L}_{5}[W, C]\right)$. By Theorem 4.1, as $I$ is semi-precompact, $\Phi$ is semiprecompact if and only if $S$ is semi-precompact. But $S^{\prime}(f)=f S$ and hence $S^{\prime}=\Phi$, which gives us the required result.

5. Applications to collectively semi-precompact sets of continuous linear operators. We use the concept of collective semiprecompactness of a set $\mathscr{H} \subset \mathscr{L}[X, Y]$ as stated in Definition 1.2. Throughout this section, $X$ and $Y$ denote locally convex Hausdorff spaces.

The following three lemmas are results which are known in other forms but are stated and proved here because of lack of references.

Lemma 5.1. Let $Y$ be a locally convex Hausdorff space. The sets

$$
G_{q}=\left\{g \in Y^{\prime}:|g(y)| \leqq q(y) \text { for all } y \in Y\right\}
$$

form a fundamental system of $\beta\left(Y^{\prime}, Y\right)$-bounded sets in $Y^{\prime}$, where $q$ runs over the continuous seminorms on $Y$, if and only if $Y$ is infrabarrelled.

Proof. Suppose the $G_{q}$ 's form a fundamental system of $\beta\left(Y^{\prime}, Y\right)$ bounded sets in $Y^{\prime}$. Each $G_{q}$ is equicontinuous [18, p. 200] and hence, as every subset of an equicontinuous set is equicontinuous, each $\beta\left(Y^{\prime}, Y\right)$-bounded set in $Y^{\prime}$ is equicontinuous. This implies that $Y$ is infrabarrelled by Lemma 4.2.

Conversely, suppose $Y$ is infrabarrelled. Then, by Lemma 4.2, if $M_{Y^{\prime}}$ is $\beta\left(Y^{\prime}, Y\right)$-bounded in $Y^{\prime}$, it is equicontinuous. By [18, p. 200], there exists a continuous seminorm $q$ on $Y$ such that

$$
M_{Y}, \subset G_{q} .
$$

The result follows.

Lemma 5.2. Let $Y$ be a locally convex Hausdorff space which is infrabarrelled. Let $M_{Y}$, be a $\beta\left(Y^{\prime}, Y\right)$-bounded subset of $Y^{\prime}$. Then the function

$$
q(y)=\sup _{g \in M_{Y^{\prime}}}|g(y)|
$$

is a continuous seminorm on $Y$.

Proof. As $M_{Y^{\prime}}$ is $\beta\left(Y^{\prime}, Y\right)$-bounded and $Y$ is infrabarrelled, $M_{Y^{\prime}}$ 
is equicontinuous. Thus the polar of $M_{Y^{\prime}}$ in $Y$,

$$
M_{Y^{\prime}}^{\circ}=\left\{y \in Y:|g(y)| \leqq 1 \text { for all } g \in M_{Y^{\prime}}\right\} \text {, }
$$

is a neighborhood of zero in $Y$, for, as $M_{Y^{\prime}}$ is equicontinuous, there exists a neighborhood $V_{Y}$ of zero in $Y$ such that $\left|M_{Y^{\prime}}\left(V_{Y}\right)\right| \leqq 1$ and obviously $M_{Y^{\prime}}^{\circ} \supset V_{Y}$. But

$$
\begin{aligned}
M_{Y^{\prime}}^{\circ} & =\left\{y \in Y: \sup _{g \in M_{Y^{\prime}}}|g(y)| \leqq 1\right\} \\
& =\{y \in Y: q(y) \leqq 1\} .
\end{aligned}
$$

As $q$ is obviously a seminorm and is continuous by the last statement, the result follows.

The following lemma is an interesting characterization of infrabarrelled spaces in terms of the adjoint mapping.

Lemma 5.3. Let $X, Y$ be locally convex Hausdorff spaces. Then the adjoint mapping

$$
A: \mathscr{L}_{\varpi}[X, Y] \longrightarrow \mathscr{L}_{\varpi}\left[Y^{\prime}, X^{\prime}\right],
$$

defined by $A(T)=T^{\prime}$, is a continuous linear map for all $X$, where $X^{\prime}, Y^{\prime}$ have their $\beta\left(X^{\prime}, X\right), \beta\left(Y^{\prime}, Y\right)$ topologies respectively, if and only if $Y$ is infrabarrelled.

Proof. The adjoint mapping $A$ is well defined by [18, Proposition 3, Corollary, p. 256].

Assume that $A$ is continuous for every locally convex Hausdorff space $X$. Then, in particular, it is true for $X=C$. Thus the map

$$
A: \mathscr{L}_{\varpi}[C, Y] \longrightarrow \mathscr{L}_{\mathfrak{b}}\left[Y^{\prime}, C^{\prime}\right]
$$

is continuous. Now $C^{\prime}$ is isomorphic to $C$ and hence $\mathscr{L}_{5}\left[Y^{\prime}, C^{\prime}\right]$ is topologically isomorphic to $Y^{\prime \prime}$ with its $\beta\left(Y^{\prime \prime}, Y^{\prime}\right)$ topology. Let

$$
\phi: \mathscr{L}_{b}\left[Y^{\prime}, C^{\prime}\right] \longrightarrow Y^{\prime \prime}
$$

be the isomorphism. We can identify $\mathscr{L}_{\varpi}[C, Y]$ with $Y$ itself by the mapping

$$
\psi: Y \longrightarrow \mathscr{L}_{\S}[C, Y],
$$

where $\psi(y)(\alpha)=\alpha y$ for each $\alpha \in C$. It is easy, but tedious, to prove that $\psi$ is also a topological isomorphism. We have the following situation

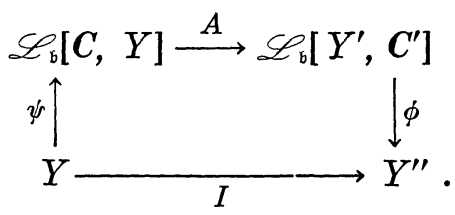


By defining $\phi$ in the obvious way, namely

$$
\phi(T)\left(y^{\prime}\right)=T\left(y^{\prime}\right)(1),
$$

where $T \in \mathscr{L}_{5}\left[Y^{\prime}, C^{\prime}\right]$ and $y^{\prime} \in Y^{\prime}$, and letting $I$ be the evaluation mapping of $Y \rightarrow Y^{\prime \prime}$, defined by $I(y)\left(y^{\prime}\right)=y^{\prime}(y)$, the above diagram commutes. Hence $I=\phi \circ A \circ \psi$. But $\phi, A, \psi$ are continuous and hence $I$ is continuous. This implies that $Y$ is infrabarrelled [21, Proposition 20.4(i), p. 192].

Conversely, assume $Y$ is infrabarrelled. As $X^{\prime}$ has its strong topology, its continuous seminorms are given by

$$
r_{M_{X}}(f)=\sup _{x \in M_{X}}|f(x)|
$$

where $f \in X^{\prime}$ and $M_{X}$ is bounded in $X$. Thus the continuous seminorms on $\mathscr{L}_{b}\left[Y^{\prime}, X^{\prime}\right]$ are given by

$$
R_{M_{X}, M_{Y^{\prime}}}\left(T^{\prime}\right)=\sup _{g \in M_{Y^{\prime}}} r_{M_{X}}\left(T^{\prime}(g)\right),
$$

where $M_{X}$ is a bounded subset of $X$ and $M_{Y^{\prime}}$ is a $\beta\left(Y^{\prime}, Y\right)$-bounded subset of $Y^{\prime}$. Hence, by definition,

$$
R_{M_{X}, M_{Y^{\prime}}}\left(T^{\prime}\right)=\sup _{g \in M_{Y^{\prime}}} \sup _{x \in M_{X}}\left|\left[T^{\prime}(f)\right](x)\right| \text {. }
$$

Hence a basic neighborhood of zero in $\mathscr{L}_{5}\left[Y^{\prime}, X^{\prime}\right]$ is

$$
\mathscr{V}^{\prime}=\left\{T^{\prime} \in \mathscr{L}\left[Y^{\prime}, X^{\prime}\right]: R_{M_{X}, M_{Y^{\prime}}}\left(T^{\prime}\right) \leqq 1\right\} .
$$

As $Y$ is infrabarrelled, $M_{Y}$, is equicontinuous and by Lemma 5.2

$$
q(y)=\sup _{g \in M_{Y}^{\prime}}|g(y)|
$$

is a continuous seminorm on $Y$. Let

$$
\mathscr{V}=\left\{T \in \mathscr{L}[X, Y]: q_{M_{X}}(T)=\sup _{x \in M_{X}} q(T(x)) \leqq 1\right\} .
$$

This is a neighborhood of zero in $\mathscr{L}_{5}[X, Y]$. We claim that $A(\mathscr{V}) \subset \mathscr{V}^{\prime}$, implying that $A$ is continuous (for it is obviously linear). Let $T \in \mathscr{C}$. Then $q_{M_{X}}(T) \leqq 1$ and, since $\sup _{x \in M_{X}} q(T(x))=\sup _{x \in M_{X}} \sup _{g \in M_{Y^{\prime}}}|g(T(x))|$ by definition of $q$, we have $q_{M_{X}}(T)=\sup _{g \in M_{Y^{\prime}}} \sup _{x \in M_{X}}|g(T(x))|=$ $\sup _{g \in M_{Y^{\prime}}} \sup _{x \in M_{X}}\left|\left[T^{\prime}(g)\right](x)\right|=R_{M_{X}, M_{Y^{\prime}}}\left(T^{\prime}\right) \leqq 1$. Thus $A(T)=T^{\prime} \in \mathscr{V}^{\prime}$ and the result follows.

The next three theorems generalize some results of Palmer [25] to locally convex Hausdorff spaces (as any Banach space is barrelled and hence infrabarrelled).

THEOREM 5.1. Let $X$ and $Y$ be locally convex Hausdorff spaces 
with $Y$ infrabarrelled. Then a subset $\mathscr{H} \subset \mathscr{L}_{5}[X, Y]$ is a precompact set of semi-precompact operators if and only if

(1) $\mathscr{H}$ is collectively semi-precompact,

(2) $\mathscr{H}^{\prime}\left(y^{\prime}\right)=\left\{H^{\prime}\left(y^{\prime}\right): H \in \mathscr{H}\right\}$ is precompact for all $y^{\prime} \in Y^{\prime}$.

Proof. Suppose $\mathscr{H}$ is a precompact set of semi-precompact operators. The proof of (1) is incorporated, in a slightly different form, in Proposition 2.3 of [14], however, for the sake of completeness we utilize the previous results to prove it.

It is obvious that $\mathscr{H} \subset \mathscr{K}_{5}[X, Y]$ and is precompact therein. By Theorem 3.1, $\left.\mathscr{H}\right|_{H_{X}}$ has equal variation on $M_{X}$ for every bounded subset $M_{X}$ of $X$ and $\mathscr{H}(x)$ is precompact for all $x \in X$. Let $M_{X}$ be a bounded subset of $X$. We prove that $\mathscr{H}\left(M_{X}\right)$ is precompact. Let $V_{Y}$ be a basic neighborhood of zero in $Y$. Then, as $\left.\mathscr{H}\right|_{M_{X}}$ has equal variation on $M_{X}$, there exists a finite cover $M_{1}, \cdots, M_{n}$ of $M_{X}$ such that

$$
H\left(M_{i}\right)-H\left(M_{i}\right) \subset U_{Y} \quad i=1, \cdots, n, \quad H \in \mathscr{H},
$$

where $U_{Y}$ is a neighborhood of zero in $Y$ such that $U_{Y}+U_{Y} \subset V_{Y}$. Assume without loss of generality that the $M_{i}$ are nonempty. Let $x_{i} \in M_{i}$ for $i=1, \cdots, n$. Then $\mathscr{C}\left(x_{i}\right)$ is precompact for $i=1, \cdots, n$. As in the proof of Theorem 2.1, we define the mappings

$$
\Theta_{x_{i}}: \mathscr{H} \longrightarrow Y
$$

by $\Theta_{x_{i}}(H)=H\left(x_{i}\right)$. These are precompact by hypothesis and, by Lemma 2.2, $\left\{\Theta_{x_{1}}, \cdots, \Theta_{x_{n}}\right\}$ has equal variation on $\mathscr{H}$. Thus there exists a finite cover $\mathscr{H}_{1}, \cdots, \mathscr{H}_{m}$ of $\mathscr{H}$ such that

$$
\mathscr{H}_{j}\left(x_{i}\right)-\mathscr{H}_{j}\left(x_{i}\right) \subset U_{Y} \quad i=1, \cdots, n ; j=1, \cdots, m .
$$

Assume without loss of generality that the $\mathscr{H}_{j}$ are nonempty and let $H_{j} \in \mathscr{H}_{j}$ for $j=1, \cdots, m$. Then we claim that

$$
\mathscr{H}\left(M_{X}\right) \subset \bigcup_{j=1}^{m} \bigcup_{i=1}^{n} H_{j}\left(x_{i}\right)+V_{Y},
$$

implying that $\mathscr{H}\left(M_{X}\right)$ is precompact. For let $H(x) \in \mathscr{H}\left(M_{X}\right)$. Then there exists an $M_{i}$ such that $x \in M_{i}$ and an $\mathscr{H}_{j}$ such that $H \in \mathscr{H}_{j}$. Now

$$
H(x)-H_{j}\left(x_{i}\right)=\left(H(x)-H\left(x_{i}\right)\right)+\left(H\left(x_{i}\right)-H_{j}\left(x_{i}\right)\right) \in U_{Y}+U_{Y} \subset V_{Y}
$$

or $H(x) \in H_{j}\left(x_{i}\right)+V_{Y}$. This proves (1). By Lemma 5.3, the adjoint mapping is continuous and linear in this case. Hence it is uniformly continuous and, as $\mathscr{H}$ is precompact, $\mathscr{H}^{\prime}=A(\mathscr{H})$ is precompact in $\mathscr{L}_{\mathrm{b}}\left[Y^{\prime}, X^{\prime}\right]$. By the corollary to Theorem 4.1 (Schauder's theorem), 
the operators in $\mathscr{H}^{\prime}$ are semi-precompact. Hence $\mathscr{H}^{\prime}$ is a precompact set of semi-precompact operators. By Theorem $3.1, \mathscr{H}^{\prime}\left(y^{\prime}\right)$ is precompact for all $y^{\prime} \in Y^{\prime}$. Thus conditions (1) and (2) hold.

Conversely, assume conditions (1) and (2) hold. Let $\mathscr{V}\left(M_{X}, q\right)$, defined by

$$
\mathscr{Y}\left(M_{X}, q\right)=\left\{T \in \mathscr{L}[X, Y]: q_{M_{X}}(T) \leqq 1\right\},
$$

where $M_{X}$ is bounded in $X$ and $q$ is a continuous seminorm on $Y$, be an arbitrary basic neighborhood of zero in $\mathscr{L}_{b}[X, Y]$. Now we know that

$$
G_{q}=\left\{g \in Y^{\prime}:|g(y)| \leqq q(y) \text { for all } y \in Y\right\}
$$

is an equicontinuous set of linear functionals on $Y$. By (1), $\mathscr{H}\left(M_{X}\right)$ is precompact. An equicontinuous set of linear functionals is uniformly equicontinuous and, as $G_{q}$ is equicontinuous, $G_{q}(y)$ is bounded and hence precompact in $C$ for each $y \in Y$. Thus, by corollary to Proposition $2.1, G_{q}$ is a precompact subset of $\mathscr{K}\left(\mathscr{H}\left(M_{X}\right), C\right)$. This implies that there exists a finite subset $\left\{g_{1}, \cdots, g_{n}\right\} \subset G_{q}$ such that

$$
\left.\left.G_{q}\right|_{\mathscr{C}\left(M_{X}\right)} \subset \bigcup_{i=1}^{n} g_{i}\right|_{\mathscr{C}\left(M_{X}\right)}+\mathscr{B}(0,1 / 3),
$$

where $\mathscr{B}(0,1 / 3)$ is the neighborhood of zero in $\mathscr{C}\left(\mathscr{H}\left(M_{X}\right), C\right)$ defined by

$$
\mathscr{B}(0,1 / 3)=\left\{g \in \mathscr{C}\left(\mathscr{H}\left(M_{X}\right), C\right): \sup _{y \in \mathscr{C}\left(M_{X}\right)}|g(y)| \leqq 1 / 3\right\} .
$$

Let $V_{X^{\prime}}$ be the $\beta\left(X^{\prime}, X\right)$-neighborhood of zero in $X^{\prime}$ given by

$$
V_{X^{\prime}}=\left\{f \in X^{\prime}: \sup _{x \in M_{X}}|f(x)| \leqq 1 / 3\right\} .
$$

By hypothesis (2), $\mathscr{H}^{\prime}\left(g_{i}\right)$ is precompact for $i=1, \cdots, n . H^{\prime}\left(g_{i}\right)=$ $g_{i} H$ for all $H \in \mathscr{H}$, by definition, and hence the $g_{i}$ can be considered as precompact mappings from $\mathscr{H}$ into $X^{\prime}$. By Lemma 2.2, the $g_{i}$ have equal variation on $\mathscr{H}$, which means there exists a finite cover $\mathscr{H}_{1}, \cdots, \mathscr{H}_{m}$ of $\mathscr{H}$ such that

$$
g_{i} \mathscr{H}_{j}-g_{i} \mathscr{H}_{j} \subset V_{X^{\prime}} \quad i=1, \cdots, n ; j=1, \cdots, m .
$$

Let $H_{j} \in \mathscr{H}_{j}$ for $j=1, \cdots, m$, again assuming, without loss of generality, that the $\mathscr{H}_{j}$ 's are nonempty. We now claim that

$$
\mathscr{H} \subset \bigcup_{j=1}^{m} H_{j}+\mathscr{Y}\left(M_{X}, q\right),
$$

implying that $\mathscr{H}$ is precompact. ( $\mathscr{H}$ obviously consists of semiprecompact operators by (1).) If $H \in \mathscr{H}$, then $H \in \mathscr{H}_{j}$ for some $j \in\{1, \cdots, m\}$. We show that $H-H_{j} \in \mathscr{Y}\left(M_{X}, q\right)$. Let $x \in M_{X}$ and 
$g \in G_{q} . \quad$ As $\left.\left.G_{q}\right|_{\mathscr{C}\left(M_{X}\right)} \subset \bigcup_{i=1}^{n} g_{i}\right|_{\mathscr{\mathscr { C }}\left(M_{X}\right)}+\mathscr{B}(0,1 / 3)$, there exists a $g_{i}$, $i \in\{1, \cdots, n\}$, such that

$$
\sup _{y \in \mathscr{C}\left(M_{X}\right)}\left|g_{i}(y)-g(y)\right| \leqq 1 / 3 .
$$

Thus

$$
\begin{aligned}
\left|g\left(H-H_{j}\right)(x)\right| & =\left|g H(x)-g H_{j}(x)\right| \\
& =\left|g H(x)-g_{i} H(x)+g_{i} H(x)-g_{i} H_{j}(x)+g_{i} H_{j}(x)-g H_{j}(x)\right| \\
& \leqq \\
& \left.\leqq 1 / 3+g_{i}\right) H(x)|+|\left(g_{i} H-g_{i} H_{j}\right)(x)|+|\left(g_{i}-g\right) H_{j}(x) \mid \\
&
\end{aligned}
$$

which means that for all $x \in M_{X}, g \in G_{q}$

$$
\left|g\left(H-H_{j}\right)(x)\right| \leqq 1 .
$$

By the Hahn-Banach theorem [24, Proposition 8, p. 191], for each $x \in M_{X}$ there exists a $g \in G_{q}$ such that

$$
g\left(\left(H-H_{j}\right)(x)\right)=q\left(\left(H-H_{j}\right)(x)\right) .
$$

Hence $q\left(\left(H-H_{j}\right)(x)\right) \leqq 1$ for all $x \in M_{X}$ or $q_{M_{X}}\left(H-H_{j}\right) \leqq 1$, which implies that $H-H_{j} \in \mathscr{Y}\left(M_{X}, q\right)$. This proves the converse statement. 2.2].

We now give the essentially dual theorem as in [25, Theorem

THEOREM 5.2. Let $X$ and $Y$ be locally convex Hausdorff spaces with $Y$ infrabarrelled. Then a subset $\mathscr{H} \subset \mathscr{L}_{5}[X, Y]$ is a precompact set of semi-precompact operators if and only if

(1) $\mathscr{H}(x)$ is precompact for all $x \in X$,

(2) $\mathscr{H}^{\prime}$ is collectively semi-precompact.

Proof. The theorem can be proved using similar arguments to those in the proof of Theorem 5.1. However, we use Theorem 5.1 for our proof.

Assume $\mathscr{H}$ is a precompact set of semi-precompact operators. By Theorem 5.1, $\mathscr{H}(x)$ is precompact for all $x \in X$ as each $\{x\}$ is bounded. This proves (1). As the adjoint mapping $A$ is continuous and linear, $A(\mathscr{H})=\mathscr{H}^{\prime}$ is precompact in $\mathscr{L}_{6}\left[Y^{\prime}, X^{\prime}\right]$ and consists of semi-precompact operators by corollary to Theorem 4.1. Using Theorem 5.1 again, $\mathscr{H}^{\prime}$ is collectively semi-precompact, proving (2).

Conversely, assume (1) and (2) hold. By (2), each $H^{\prime} \in \mathscr{H}^{\prime}$ is semi-precompact and hence, by corollary to Theorem 4.1, each $H \in \mathscr{H}$ is semi-precompact. We prove that (1) and (2) imply $\left.\mathscr{H}\right|_{M_{X}}$ has equal variation on $M_{X}$ for all bounded $M_{X} \subset X$, for then the result holds by Theorem 3.1, as a subset of $\mathscr{K}[X, Y]$ is precompact in 
$\mathscr{K}_{5}[X, Y]$ if and only if it is precompact in $\mathscr{L}_{5}[X, Y]$ (as $\mathscr{K}_{5}[X, Y]$ has the relative topology). Let $V_{Y}=\{y \in Y: q(y) \leqq 1\}$ be an arbitrary basic neighborhood of zero in $Y$, where $q$ is a continuous seminorm on $Y$. Let $M_{X} \subset X$ be bounded.

$$
G_{q}=\left\{g \in Y^{\prime}:|g(y)| \leqq q(y) \text { for all } y \in Y\right\}
$$

is equicontinuous and hence $\beta\left(Y^{\prime}, Y\right)$-bounded in $Y^{\prime}$. Thus, by (2), $\mathscr{H}^{\prime}\left(G_{q}\right)=G_{q} \mathscr{H}$ is precompact in $X^{\prime}$, where $X^{\prime}$ has its strong topology and coincides with the space $\mathscr{L}_{5}[X, C]$. Each element of $G_{q} \mathscr{H}$ is obviously a semi-precompact operator. Hence, by Theorem 3.1, $\left.G_{q} \mathscr{H}\right|_{M_{X}}$ has equal variation on $M_{X}$. We thus have a finite cover $M_{1}, \cdots, M_{n}$ of $M_{X}$ such that

$$
\left|g H\left(M_{i}\right)-g H\left(M_{i}\right)\right| \leqq 1 \quad i=1, \cdots, n, g \in G_{q}, H \in \mathscr{H} .
$$

If $x, y \in M_{i}$, we have

$$
|g H(x)-g H(y)| \leqq 1 \quad g \in G_{q}, H \in \mathscr{H}
$$

or

$$
|g(H(x)-H(y))| \leqq 1 \quad g \in G_{q}, H \in \mathscr{H} .
$$

But by the Hahn-Banach theorem [24, Proposition 8, p. 191], as $H(x)-H(y) \in Y$, we know there exists a $g \in G_{q}$ such that

$$
g(H(x)-H(y))=q(H(x)-H(y)) .
$$

This says that $q(H(x)-H(y)) \leqq 1$ for all $x, y \in M_{i}, H \in \mathscr{H}$, which implies that

$$
H\left(M_{i}\right)-H\left(M_{i}\right) \subset V_{Y} \quad i=1, \cdots, n, H \in \mathscr{H} .
$$

Then, by definition, $\left.\mathscr{H}\right|_{M_{X}}$ has equal variation on $M_{X}$. We have proved now that $\mathscr{H}$ is a precompact set of semi-precompact operators.

As a direct consequence of Theorems 5.1 and 5.2, we have the generalization of Palmer's result [25, Theorem 3.1] to locally convex Hausdorff spaces.

THeOREM 5.3. Let $X, Y$ be locally convex Hausdorff spaces with $Y$ infrabarrelled. Then a subset $\mathscr{H} \subset \mathscr{L}_{b}[X, Y]$ is a precompact set of semi-precompact operators if and only if

(1) $\mathscr{H}$ is collectively semi-precompact,

(2) $\mathscr{H}^{\prime}$ is collectively semi-precompact.

Proof. This is trivial using the more strict characterizations of Theorems 5.1 and 5.2. 
Added in proof. Since submitting this article the author has learned that some of his results on characterizations of precompactness using the notion of equal variation and on generalizing Schauder's theorem have also been obtained, independently, by $\mathrm{H}$. Apiola ("On the tensorproduct and product $\operatorname{Hom}(f, g)$ of compact operators in locally convex topological vector spaces", Ann. Acad. Sci. Fenn. Ser. A. I. Math., 544 (1973), 33pp.).

\section{REFERENCES}

1. J. C. Alexander, Compact Banach algebras, Proc. London Math. Soc., (3) 18 (1968), $1-18$.

2. P. M. Anselone, Collectively compact and totally bounded sets of linear operators,

J. Math. Mech., 17 (1968), 613-621.

3. - Compactness properties of sets of operators and their adjoints, Math. Z., 113 (1970), 233-236.

4. - Collectively Compact Operator Approximation Theory and Applications to Integral Equations, Prentice-Hall, Englewood Cliffs, 1971.

5. P. M. Anselone and R. H. Moore, Approximate solutions of integral and operator equations, J. Math. Anal. Appl., 9 (1964), 268-277.

6. P. M. Anselone and T. W. Palmer, Collectively compact sets of linear operators, Pacific J. Math., 25 (1968), 417-422.

7. - Spectral analysis of collectively compact, strongly convergent operator sequences, Pacific J. Math., 25 (1968), 423-431.

8. - Spectral properties of collectively compact sets of linear operators, J. Math. Mech., 17 (1968), 853-860.

9. R. G. Bartle, On compactness in functional analysis, Trans. Amer. Math. Soc., 79 (1955), 35-57.

10. F. F. Bonsall, Compact operators from an algebraic standpoint, Glasgow Math. J., 8 (1967), 41-49.

11. J. D. DePree and J. A. Higgins, Collectively compact sets of linear operators, Math.

Z., 115 (1970), 366-370.

12. J. D. DePree and H. S. Klein, Characterizations of collectively compact sets of linear operators, Pacific J. Math., (To appear).

13. - Semigroups and collectively compact sets of linear operators, Pacific J. Math., (To appear).

14. M. V. Deshpande and N. E. Joshi, Collectively compact and semi-compact sets of linear operators in topological vector spaces, Pacific J. Math., 43 (1972), 317-326.

15. N. Dunford and J. T. Schwartz, Linear Operators, Part I, Interscience, New York, 1958.

16. S. Goldberg, Unbounded Linear Operators, McGraw-Hill, New York, 1966.

17. A. Grothendieck, Sur les applications lineaires faiblement compactes d'espaces du type $C(K)$, Canad. J. Math., 5 (1953), 129-173.

18. J. Horváth, Topological Vector Spaces and Distributions, Volume I, AddisonWesley, Reading, Mass., 1966.

19. S. Kakutani, A proof of Schauder's theorem, J. Math. Soc. Japan, 3 (1951), 228-231. 20. J. L. Kelley, General Topology, Van Nostrand, Princeton, 1955.

21. J. L. Kelley, I. Namioka, and Co-authors, Linear Topological Spaces, Van Nostrand, Princeton, 1963.

22. H. S. Klein, Collectively Compact Sets of Linear Operators, Ph. D. Thesis, New Mexico State University, 1972. 
23. G. Köthe, Zur Theorie der kompakten Operatoren in lokalkonvexen Räumen, Portugal. Math., 13 (1954), 97-104.

24. - Topological Vector Spaces I, Springer-Verlag, Berlin $\cdot$ Heidelberg $\cdot$ New York, 1969.

25. T. W. Palmer, Totally bounded sets of precompact linear operators, Proc. Amer. Math. Soc., 20 (1969), 101-106.

26. H. Poppe, Zum Satz von Ascoli und Arzelà, Math. Ann., 171 (1967), 46-53.

27. J. R. Ringrose, Complete continuity conditions on linear operators, Proc. London Math. Soc., (3) 8 (1958), 343-356.

28. A. P. Robertson and W. J. Robertson, Topological Vector Spaces, Cambridge University Press, London, 1964.

29. H. H. Schaefer, Topological Vector Spaces, Macmillan, New York, 1966.

30. J. Schauder, $\ddot{U} b e r$ lineare, vollstetige Funktionaloperationen, Studia Math., 2 (1930), 183-196.

31. F. Treves, Topological Vector Spaces, Distributions and Kernels, Academic Press, New York, 1967.

32. K. Vala, On compact sets of compact operators, Ann. Acad. Sci. Fenn. Ser. A. I. Math., 351 (1964), 9 pp.

Received July 30, 1973 and in revised form February 8, 1974.

The Flinders University of South Australia 



\section{PACIFIC JOURNAL OF MATHEMATICS}

EDITORS

RICHARD ARens (Managing Editor)

University of California

Los Angeles, California 90024

\section{R. A. Beaumont}

University of Washington

Seattle. Washington 98105

\section{J. DugundjI}

Department of Mathematics University of Southern California Los Angeles, California 90007

D. Gilbarg and J. Milgram

Stanford University

Stanford. California 94305

\section{ASSOCIATE EDITORS}
E. F. BECKENBACH
B. H. NEUMANN
F. WOLF
K. Yoshida

\section{SUPPORTING INSTITUTIONS}

\author{
UNIVERSITY OF BRITISH COLUMBIA \\ CALIFORNIA INSTITUTE OF TECHNOLOGY \\ UNIVERSITY OF CALIFORNIA \\ MONTANA STATE UNIVERSITY \\ UNIVERSITY OF NEVADA \\ NEW MEXICO STATE UNIVERSITY \\ OREGON STATE UNIVERSITY \\ UNIVERSITY OF OREGON \\ OSAKA UNIVERSITY
}

\author{
UNIVERSITY OF SOUTHERN CALIFORNIA \\ STANFORD UNIVERSITY \\ UNIVERSITY OF TOKYO \\ UNIVERSITY OF UTAH \\ WASHINGTON STATE UNIVERSITY \\ UNIVERSITY OF WASHINGTON

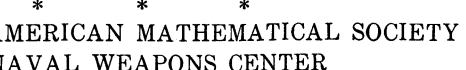

The Supporting Institutions listed above contribute to the cost of publication of this Journal, but they are not owners or publishers and have no responsibility for its content or policies.

Mathematical papers intended for publication in the Pacific Journal of Mathematics should be in typed form or offset-reproduced, (not dittoed), double spaced with large margins. Underline Greek letters in red, German in green, and script in blue. The first paragraph or two must be capable of being used separately as a synopsis of the entire paper. Items of the bibliography should not be cited there unless absolutely necessary, in which case they must be identified by author and Journal, rather than by item number. Manuscripts, in duplicate if possible, may be sent to any one of the four editors. Please classify according to the scheme of Math. Rev. Index to Vol. 39. All other communications to the editors should be addressed to the managing editor, or Elaine Barth, University of California, Los Angeles, California, 90024.

100 reprints are provided free for each article, only if page charges have been substantially paid Additional copies may be obtained at cost in multiples of 50 .

The Pacific of Journal Mathematics is issued monthly as of January 1966. Regular subscription rate: $\$ 72.00$ a year (6 Vols., 12 issues). Special rate: $\$ 36.00$ a year to individual members of supporting institutions.

Subscriptions, orders for back numbers, and changes of address should be sent to Pacific Journal of Mathematics, 103 Highland Boulevard, Berkeley, California, 94708.

\section{PUBLISHED BY PACIFIC JOURNAL OF MATHEMATICS, A NON-PROFIT CORPORATION}

Printed at Kokusai Bunken Insatsusha (International Academic Printing Co., Ltd.), 270, 3-chome Totsuka-cho. Shinjuku-ku, Tokyo 160. Japan.

Copyright (C) 1973 by Pacific Journal of Mathematics Manufactured and first issued in Japan 


\section{Pacific Journal of Mathematics}

\section{Vol. 52, No. $2 \quad$ February, 1974}

Harm Bart, Spectral properties of locally holomorphic vector-valued functions .....

J. Adrian (John) Bondy and Robert Louis Hemminger, Reconstructing infinite

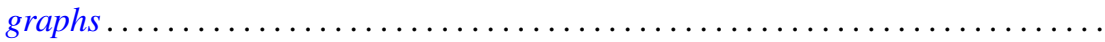

Bryan Edmund Cain and Richard J. Tondra, Biholomorphic approximation of planar

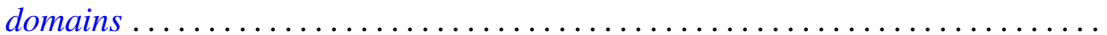

Richard Carey and Joel David Pincus, Eigenvalues of seminormal operators,

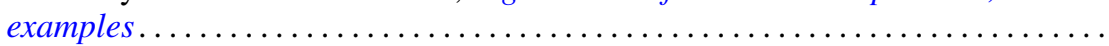

Tyrone Duncan, Absolute continuity for abstract Wiener spaces . . . . . . . . . . . . Joe Wayne Fisher and Louis Halle Rowen, An embedding of semiprime

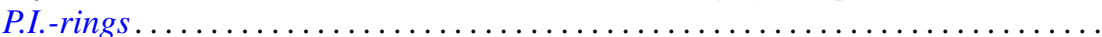

Andrew S. Geue, Precompact and collectively semi-precompact sets of semi-precompact continuous linear operators. . . . . . . . . . . . . . . .

Charles Lemuel Hagopian, Locally homeomorphic $\lambda$ connected plane continua ..... . Darald Joe Hartfiel, A study of convex sets of stochastic matrices induced by

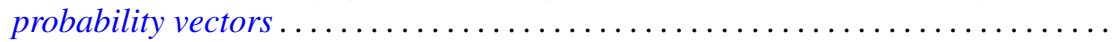

Yasunori Ishibashi, Some remarks on high order derivations $\ldots \ldots \ldots \ldots \ldots \ldots \ldots$ Donald Gordon James, Orthogonal groups of dyadic unimodular quadratic forms.

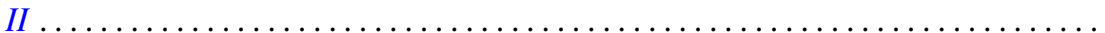

Geoffrey Thomas Jones, Projective pseudo-complemented semilattices . . . . . . . . . Darrell Conley Kent, Kelly Denis McKennon, G. Richardson and M. Schroder,

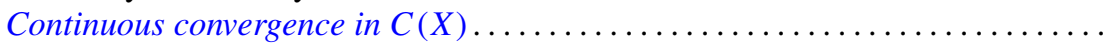

J. J. Koliha, Some convergence theorems in Banach algebras ...

Tsang Hai Kuo, Projections in the spaces of bounded linear oper

George Berry Leeman, Jr., A local estimate for typically real functions . .

475

Andrew Guy Markoe, A characterization of normal analytic spaces by the

homological codimension of the structure sheaf .........

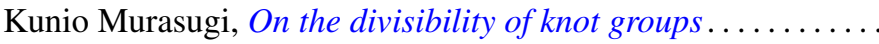

John Phillips, Perturbations of type I von Neumann algebras.

Billy E. Rhoades, Commutants of some quasi-Hausdorff matrices . .

David W. Roeder, Category theory applied to Pontryagin duality

Maxwell Alexander Rosenlicht, The nonminimality of the differential closure .

Peter Michael Rosenthal, On an inversion theorem for the general Mehler-Fock transform pair.

Alan Saleski, Stopping times for Bernoulli automorphisms

John Herman Scheuneman, Fundamental groups of compact complete locally affine complex surfaces. II. ........................

Vashishtha Narayan Singh, Reproducing kernels and operators with a cyclic vector. I. .

Peggy Strait, On the maximum and minimum of partial sums of random variables.

J. L. Brenner, Maximal ideals in the near ring of polynomials modulo 2 .

Ernst Gabor Straus, Remark on the preceding paper: "Ideals in near rings of polynomials over a field" ..........................

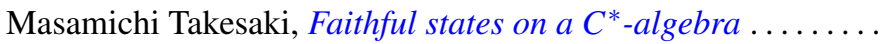

R. Michael Tanner, Some content maximizing properties of the regular simplex.

Andrew Bao-hwa Wang, An analogue of the Paley-Wiener theorem for certain

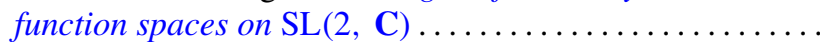

